\title{
La desigualdad ante la muerte: educación y esperanza de vida en España .
}

\author{
Miguel Requena, UNED y Grupo de Estudios 'Población y Sociedad'
}

La educación se muestra como uno de los factores discriminantes ante la muerte de la población española. Los nuevos datos del INE sobre mortalidad por edad y nivel de instrucción para hombres y mujeres permiten medir esas diferencias como probabilidades de muerte y esperanzas de vida en la España de 2012. La distancia es más que notable: un hombre de 30 años y nivel de instrucción alto puede esperar vivir de media tres años y ocho meses más que un coetáneo de nivel educativo elemental. Esa desigualdad educativa ante la muerte es mayor entre los hombres que entre las mujeres; además, la tradicional diferencia ante la mortalidad por razón de género tiende a disminuir cuanto mayor es el nivel educativo. Aunque hay que ser prudentes a la hora de establecer causalidades, todo apunta a que la educación, con sus consiguientes diferenciales en condiciones laborales, económicas y culturales, es un elemento determinante de las desigualdades ante la muerte y la longevidad.

\section{EDUCACIÓN, MORTALIDAD Y DURACIÓN DE LA VIDA COMO INDICADORES DE DESIGUALDAD}

Una copiosa literatura académica viene mostrando hace décadas la existencia de un gradiente educacional en salud con un amplio rango de implicaciones en la vida de las personas. Las desiguales tasas de mortalidad de los individuos con distintos niveles educativos y, en consecuencia, sus desiguales esperanzas de vida son una de las manifestaciones más claras de ese gradiente.

La investigación realizada hasta la fecha es muy concluyente a este respecto. En todos los casos observados, sin excepción, el logro educativo está asociado a una vida más larga: los hombres y mujeres con educación superior tienden a vivir más años que los menos educados; la vida de los hombres y mujeres con un nivel educativo bajo es más corta. Varios son los posible mecanismos por los que la educación llega a producir una mejor salud y una vida más larga: los segmentos más educados de la población disponen de más recursos económicos, pueden permitirse más y mejor atención sanitaria, disfrutan de mejores condiciones generales de vida y trabajo, desarrollan preferencias más marcadas por la buena salud y adoptan conscientemente estilos de vida más saludables gracias a que cuentan con información más valiosa sobre la salud y sus cuidados. Por todas estas razones el logro educativo tiene un alto impacto en la mejora de la salud y la prolongación de la vida individual, hasta el punto de que la OCDE (OECD 2015) atribuye las ganancias en longevidad que ha experimentado la población mundial en las últimas décadas, entre otros factores, al estilo de vida más saludable que promueve una mayor educación.

El gradiente educacional en esperanza de vida ha sido documentado en muchas sociedades desarrolladas. Pero ese tipo de evidencias no existe en España, donde - pese a lo mucho que se sabe sobre la mortalidad a lo largo del último siglo y medio (Pérez-Moreda, Reher \& SanzGimeno 2015) - aún no se han calculado tablas de mortalidad separadas para los diferentes niveles educativos. La razón de este importante déficit es obvia: lo que sabemos de las diferencias por educación procede de estudios basados en muestras locales o regionales porque hasta ahora no se disponía de los datos necesarios para estimarlas a escala nacional. Últimamente, las oficinas estadísticas regionales han comenzado a proporcionar datos, v.g., las Estadísticas Longitudinales de Supervivencia y Longevidad en Andalucía 2002-2013. Auténticos hitos en el campo son dos recientes estudios que, utilizando datos del INE no inmediatamente disponibles para su uso público, analizan las diferencias educativas de mortalidad por causas de muerte de la población española para el periodo 2001-2008 (Reques et al. 2014) y la mortalidad antes y después de la gran recesión en diferentes grupos socioeconómicos (Regidor et al. 2016). Dichos estudios no presentan, sin embargo, estimaciones de la esperanza de vida.

La reciente publicación por el Instituto Nacional de Estadística (INE) de datos de defunciones por nivel educativo y edades simples desde el año 2012 en las estadísticas vitales del Movimiento Natural de la Población (MNP) ofrece la oportunidad de calcular, por vez primera, tablas de mortalidad de periodo por educación y sexo en España. En este trabajo se presentan dichas tablas, que pueden resultar útiles por al menos tres razones. En primer lugar, los diferenciales educativos en probabilidades de muerte y esperanza de vida expresan de forma nítida y concisa una de las principales consecuencias del gradiente educacional de la salud en España. En segundo lugar, esas tablas pueden servir de base para ulteriores estimaciones de la esperanza de vida de otros segmentos, categorías, y situaciones sociales, familiares, y personales para las que la educación es un factor relevante. En tercer lugar, y al margen de su posible utilidad técnica, se espera también que los 
resultados que se presentan aquí sirvan para mitigar la mayormente injustificada valoración negativa que la opinión pública española tiende a hacer de la educación (Caínzos 2015).

\section{NUEVOS DATOS DE MORTALIDAD POR NIVEL EDUCATIVO}

Las tasas de mortalidad por edad y nivel educativo se han estimado a partir de diferentes fuentes, todas ellas suministradas por el INE y fácilmente disponibles en su página web (www.ine.es). Primero, el numerador de las tasas de mortalidad es el número de muertes por sexo y edades simples registradas por las estadísticas vitales del MNP, a las que se ha atribuido el nivel educativo con información de otras fuentes. Puesto que los boletines de defunciones no registran el nivel educativo de los fallecidos, el INE ha recuperado esa información del Padrón Municipal, el Censo de 2011 y las estadísticas de educación elaboradas por el Ministerio de Educación (INE 2015). Como el INE reconoce abiertamente, la información procedente de esas fuentes no está libre de discrepancias, que se han resuelto por medio de un algoritmo probabilístico basado en la edad y la provincia de residencia. Segundo, las proporciones de población por educación, sexo y edades simples para el año 2012 se basan también en las estimaciones del INE elaboradas a partir de aquellas mismas fuentes con los mismos procedimientos, mientras que la población clasificada por sexo y edades simples para el año 2012 se ha tomado de las Cifras de Población correspondientes al 1 de julio de 2012. De esas dos estimaciones provienen los denominadores de las tasas de mortalidad que permiten construir las tablas.
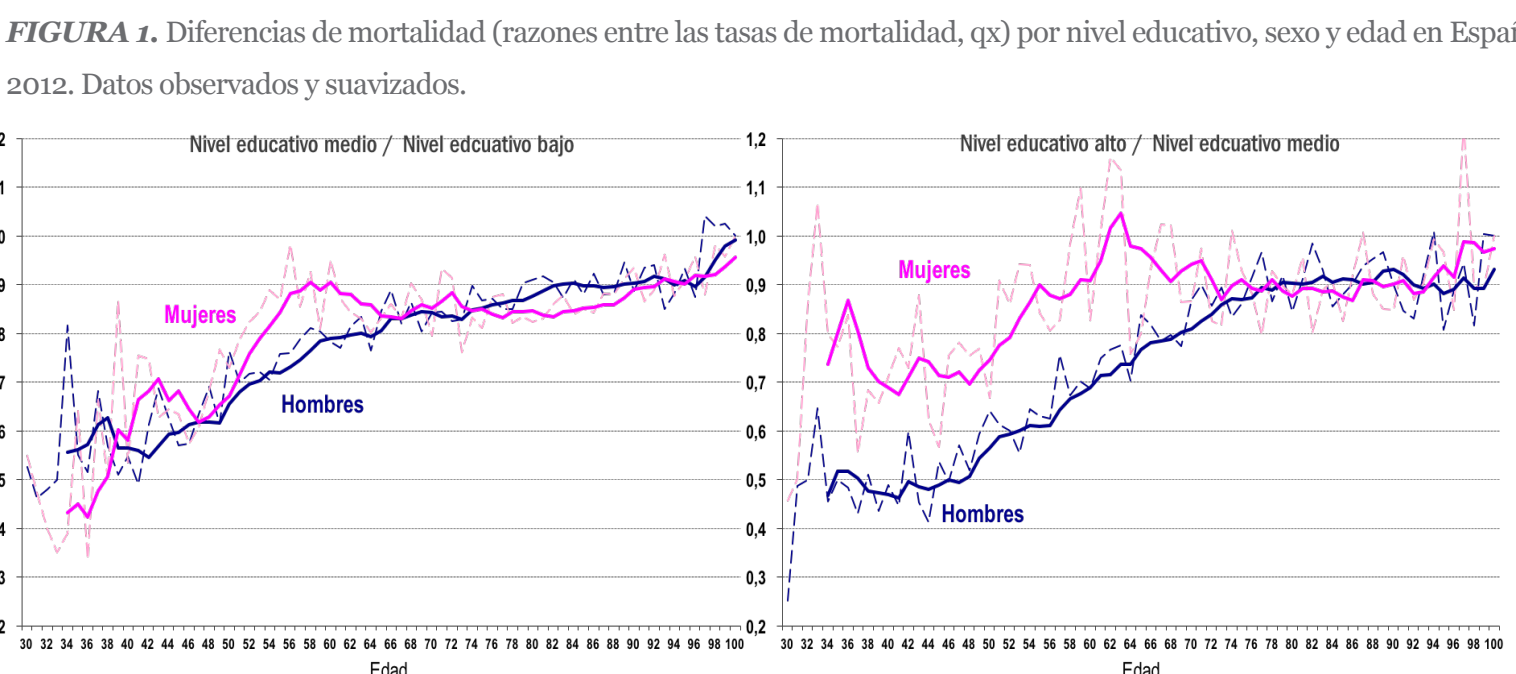

Por lo que se refiere al nivel educativo, la educación se ha agrupado en tres amplias categorías: baja educación (personas con educación primaria o inferior); educación media (personas con educación secundaria de primer y segundo ciclo y educación post-secundaria no terciaria); y educación superior (personas con titulación de grado, máster y doctorado). Aquellas muertes sin nivel educativo imputado en el MNP (2,97\% de todas las muertes en 2012) se han excluido del análisis.

A partir de esas tasas, se han construido las tablas de mortalidad por nivel educativo de la población española aplicando los procedimientos al uso a los hombres y mujeres de 30 y más años de edad en el año 2012. La elección de los 30 años como punto de arranque de la cohorte sintética que compone la tabla de periodo es apropiada porque se puede considerar que el logro educativo no varía o apenas lo hace a partir de esa edad.

\section{LA EDUCACIÓN PROTEGE CONTRA LA MORTALIDAD Y PROLONGA LA VIDA}

Las tablas de mortalidad de la población española (véanse con detalle en el apéndice) muestran importantes diferencias en mortalidad y esperanza de vida asociadas al nivel educativo. Si se comparan las razones entre las tasas de mortalidad (qx) de los niveles educativos adyacentes, se pueden apreciar notables diferenciales que sistemáticamente favorecen a los individuos con más logro educativo (Figura 1). Los cocientes entre las tasas se sitúan por debajo de la unidad a prácticamente todas las edades, lo que implica una menor propensión a morir a las mismas edades de los niveles educativos superiores cuando se los compara con los inferiores. Los 
las personas que logran sobrevivir irían igualando su probabilidad de morir con independencia de su nivel educativo; y (2) el gradiente educativo de la mortalidad es común a hombres y mujeres, pero resulta más pronunciado entre los primeros, particularmente cuando un 18,0\% y uno con educación superior sólo un 13,1\%. La educación también disminuye el riesgo de muerte de las mujeres españolas: una mujer de bajo logro educativo de 30 años tiene un 9.8\% de morir antes de los 70, frente a un 7,9\% entre las de nivel medio y solo

FIGURA 2. Esperanza de vida a distintas edades por nivel educativo y sexo. Población española en 2012

un $7.1 \%$ las de nivel superior.

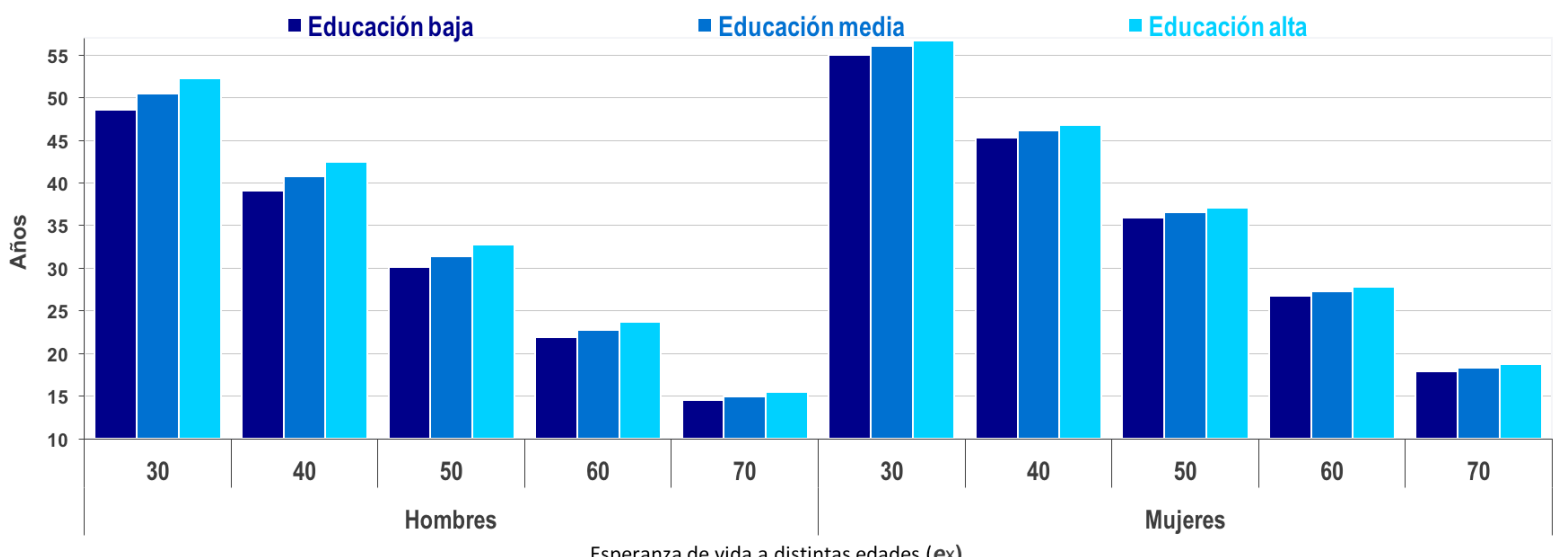

La comparación entre hombres y mujeres con el mismo nivel educativo es también ilustrativa (Figura 3). El gap de género en esperanza de vida es favorable a las mujeres cualquiera que sea el logro edu-

se comparan los niveles educativos altos con los medios.

Dados estos diferenciales, no sorprende que en España la duración de la vida de los hombres y de las mujeres esté también claramente asociada al logro educativo alcanzado (Figura 2). Los hombres de 30 años con educación superior pueden esperar vivir 52,3 años, frente a solo 50,5 años los que tienen nivel educativo medio y a 48,6 los que tienen nivel bajo. Las mujeres - con una esperanza de vida agregada para los tres niveles educativos que excede en 5,4 años a la del total de los hombres a los 30 años- también muestran esa misma asociación: mientras que las mujeres de bajo logro educativo pueden esperar vivir 55,0 años al cumplir los 30, las mujeres con nivel educativo medio alcanzarán los 56 y las tituladas superiores los 56,7. Ese mismo patrón se observa a edades más avanzadas. A los 60 años, un hombre con bajas credenciales educativas puede esperar vivir 22,0 años adicionales; si tiene titulación media, vivirá en promedio 22,8 años más; $y$, si consiguió un título universitario, su vida se podría prolongar otros 23,7. Las esperanzas a los 60 años equivalentes para las mujeres son 26,8, 27,3 y 27,8. La Figura 2 permite visualizar también que el gap educacional es mayor entre los hombres que entre las mujeres.

La misma realidad emerge al estimar el riesgo de muerte a distintas edades. Para los mismos intervalos de edad, las probabilidades de morir disminuyen con el nivel educativo. Mientras que un hombre de 30 años con bajo nivel educativo tiene un 22,8\% de probabilidades de morir antes de su 70 cumpleaños, uno de nivel medio tiene cativo; pero el diferencial se aminora a medida que el nivel educativo es más alto: a los 30 años, la ventaja relativa de las mujeres en supervivencia es de un $13.2 \%$ para los niveles educativos bajos, de un $11,0 \%$ para los medios y de solo un $8,5 \%$ para los altos. Después de los 80 años, el gap decrece para los tres niveles educativos, lo que sugiere de nuevo importantes efectos de selección.

FIGURA 3. Razones de ventaja entre esperanzas de vida (mujeres/ hombres) a distintas edades por nivel educativo

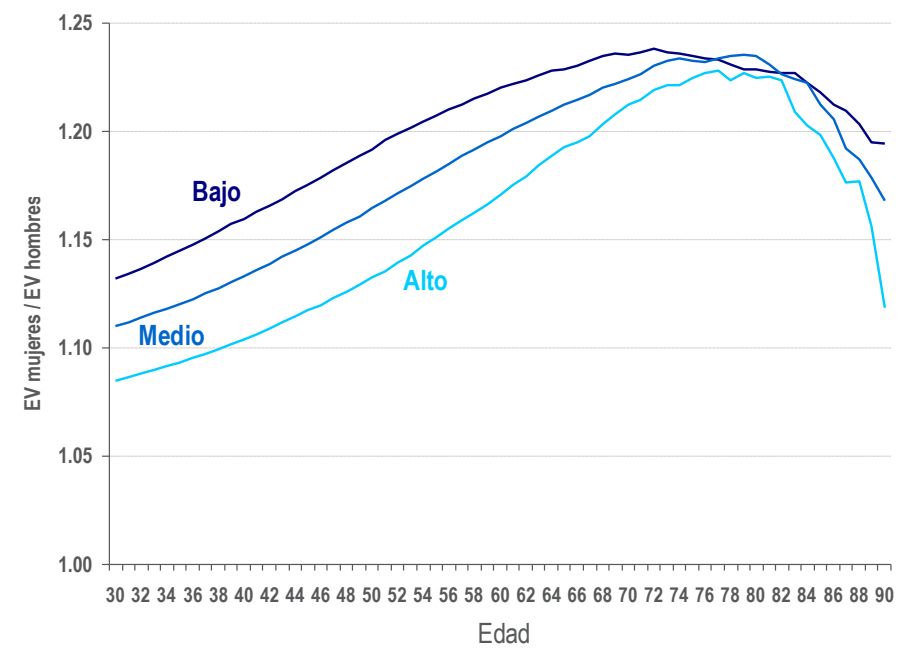

Fuente: Elaboración propia con datos del INE correspondientes al año 2012. Véase el texto para más detalles. 


\section{CONCLUSIONES Y MIRADA AL FUTURO}

Los nuevos datos españoles confirman el patrón de asociación fundamental entre supervivencia y educación que se ha observado ya abundantemente en otros países: la duración de la vida de los hombres y las mujeres españoles depende en no despreciable medida del logro educativo en el sentido de que a todas las edades las personas con más educación viven más años. Tres conclusiones merecen ser resaltadas: (1) la relación entre educación y supervivencia no parece ser lineal a lo largo de todas las edades; (2) el gap educacional es más profundo entre los hombres que entre las mujeres; y (3) el logro educativo amortigua los diferenciales en esperanza de vida entre hombres y mujeres: durante casi todo el ciclo vital, a las mismas edades, cuanto mayor el nivel de estudios alcanzado, menor el gap que separa a los hombres de las mujeres.

Sería tentador concluir este trabajo proponiendo una explicación causal de estas asociaciones tan robustas entre el logro educativo y la duración de la vida y afirmar que la educación es causa de mayor longevidad. Sin embargo, el diseño utilizado en este estudio no autoriza una lectura causal tan fuerte de los datos presentados. Por una parte, no se ha dispuesto de indicadores de ninguno de los posibles mecanismos por los cuales el logro educativo podría ser la causa de la prolongación de la vida. El hecho de que el gap educacional masculino sea mayor que el femenino sugiere que, entre otros factores, las distintas condiciones de trabajo asociadas a las diferentes categorías educativas han podido jugar un papel fundamental en la prolongación de la vida. Pero las evidencias disponibles no permiten por ahora verificar tal hipótesis. Por otra parte, hay que ser muy cuidadoso con la interpretación de las esperanzas de vida a partir de las tablas de mortalidad de periodo. Puesto que estas tablas se basan en una cohorte sintética, debe entenderse que pertenecen a una generación hipotética sometida a las condiciones de mortalidad de 2012. Los individuos observados en la tabla de mortalidad pertenecen en realidad a cohortes de nacimiento tan alejadas, y han experimentado condiciones vitales tan diferentes, que sus probabilidades de supervivencia han debido de estar sometidas a factores también muy distintos a lo largo de sus vidas.

En todo caso, los datos presentados sugieren que la educación ha jugado un papel crucial en el aumento de la supervivencia y la longevidad de los hombres y mujeres en España en las últimas décadas. Por lo tanto, cualquiera que sea el impacto causal real de la educación en la longevidad, y siempre que esta asociación básica entre educación y supervivencia no cambie, cabe esperar futuras ganancias adicionales en la esperanza de vida de la población española en la medida en que su composición educativa siga cambiando hacia un mayor nivel de educación agregado. Cabe asimismo suponer que el gap entre hombres y mujeres se hará más profundo debido a las ventajas educativas de las mujeres más jóvenes.

Hay que celebrar, en suma, la aparición y puesta a disposición del público de estos valiosos datos de defunciones por edad y nivel educativo que es de esperar se difundan pronto desagregados también por causas de muerte. Esas y otras posibles desagregaciones, incluidas desde luego las territoriales, abrirán el campo a futuros trabajos orientados a poner a prueba hipótesis más sofisticadas sobre el gradiente social de la mortalidad y a desarrollar análisis más minuciosos y profundos de las desigualdades ante la muerte en España.

Con este número empezamos una nueva serie de Perspectives. Guest Edition donde, de forma excepcional, tienen cabida la difusión de resultados de investigaciones demográficas producidas por colegas que no pertenecen al Centre d'Estudis Demogràfics, que a nuestro jucio por su interés y novedad merceden ser publicadas.

Referencias bibliográficas

Caínzos, M. (2015). La opinión pública sobre la educación en España: en tre el catastrofismo y la satisfacción. Revista Española de Sociología 23: 117-50.

INE [Instituto Nacional de Estadística] (2015). Estadística de Defunciones. Asignación de nive educativo a ficheros de defunciones de 2012, 2013 y 2014. Método de obtención y advertencias a usuarios. Madrid: Instituto Nacional de Estadística. Disponible en: http://www. ine.es $/$ jaxi $/$ menu.do? type $=$ pcax is\&path $=\% 2 \mathrm{Ft} 20 \% 2 \mathrm{Fe} 306 \&$ file $=$ ine base $\& \mathrm{~L}=0$.

OECD [Organisation for Economic Co-operation and Development] (2015). Health at a Glance 2015: OECD Indicators. Paris: OECD Publishing. Disponible en: http://dx.doi. org/10.1787/health_glance-2015-en Pérez-Moreda, V., Reher, D. \& Sanz-Gimeno, A. (2015). La conquista de la salud. Mortalidad y modernización en la España contemporánea. Madrid: Marcial Pons.

Regidor, E., Vallejo, F., Tapia Granados, J.A., Viciana-Fernández, F.J., Fuente, L., Barrio, G. (2016).
Mortality decrease according to socioeconomic groups during the economic crisis in Spain: a cohort study of 36 million people. Lancet 388 : 2642-52.

Reques, L., Giráldez-García, C., Miqueleiz, E. \& Regidor, E. (2014). Educational differences in mortality and the relative importance of different causes of death: a 7-year follow-up study of Spanish adults. Journal of Epidemiology and Community Health 68(12):1151-1160.

Editores:

Andreu Domingo y Albert Esteve
Correspondencia dirigida a:

Miguel Requena mrequena@poli.uned.es

Créditos

Edición de gráficos: Anna Turu

Maquetación

Xavier Ruiz Vilchez

Enlace url

http://ced.uab.es/es/difusion/ butlleti-perspectives-demografiques U 1 